\title{
Effect of electro spun PHB and HAP-PHB composite scaffolds characteristics on mesenchymal stem cell growth viability
}

\begin{abstract}
Cell-cell and material-cell interactions have been identified as key factors modulating cellular differentiation and extracellular matrix (ECM) production. Stem cells, In vivo and In vitro cells cultures, respond to biological signals received by their microenvironment surroundings. Via this stimulation, cells can secrete many factors to modulate their microenvironment and activate progenitor cells. This induction results in an increase in the expression of ECM proteins that are associated with cellular adhesion and proliferation and matrix mineralization. Based on the hypothesis that nanostructures can influence steam cell signalization, a long-standing goal has been fabricating a scaffold of polyhydroxybutyrate (PHB) with hydroxyapatite (HAp) incorporated to imitate a bone ECM. Using the electrospinning technique, it is possible to fabricate micro and nanostructured scaffolds. Structural characterization and viability evaluation of these scaffolds, such as adhesion and cellular proliferation, have demonstrated that scaffolds exhibit biocompatibility. Scaffolds containing HAp produce a mineralized matrix, which shows that mesenchymal stem cells differentiate to the osteogenic lineage. These scaffolds have accordingly been shown to have bone regeneration applicability in sites that are not subject to excessive loads.
\end{abstract}

Keywords: bone tissue engineering, nanostructures, hydroxyapatite, polyhydroxybutyrate, stem cells, mineralized matrix
Volume I Issue 6 - 2017

\author{
Christian Viezzer, Maria Madalena de \\ Camargo Forte, Felipe Amorim Berutti, \\ Annelise Kopp Alves, Carlos Pérez Bergmann \\ Department of Materials, Federal University of Rio Grande do \\ Sul, Brazil
}

Correspondence: Annelise Kopp Alves, Materials Department, Federal University of Rio Grande do Sul, Av. Osvaldo Aranha 99 sala 709 - 90035-190 - Porto Alegre, RS, Brazil, Tel +55 5 I 3308334I,Email annelise.alves@ufrgs.br

Received: December 08, 2017 | Published: December 29,
2017
Abbreviations: ECM, extracellular matrix; PHB, polyhydroxybutyrate; HAP, hydroxyapatite; DMF, dimethylformamide; SEM, scanning electron microscopy; TGA, thermo gravimetry; DME, dulbecco's modified eagle's Medium; FBS, fetal bovine serum; DMA, dynamic mechanic analysis.

\section{Introduction}

Repairing bone and tissue defects caused by trauma, cancer and neonatal disease is still a major challenge in medical and dental fields. Professionals in these areas are often faced with using a graft to aid the recovery of the affected areas. Grafts can be classified according to their origin as autogenous (autograft), allogeneic (from a donor of the same species) or alloplastic (biomaterials). The injured or missing tissues are typically filled with autogenous graft. ${ }^{1}$ However, the removal of an autogenous graft often results in greater morbidity to the patient, local pain and infection, and the process prolongs surgical time and increases the cost of the procedure. ${ }^{2}$ In contrast, allografts from tissue banks have been successfully used to repair tissue loss. However, using allografts is associated with limitations such as immunological compatibility, transmission of pathogens and the need for immunosuppressive drugs because of the risk of rejection. ${ }^{3}$ In recent years, the field of tissue engineering has sustained significant progress due to the emergence of nanotechnology. Some studies have indicated that the use of nanostructured features on the surface of frameworks leads to increased tissue regeneration. ${ }^{4}$ The pursuit of improving the properties of these frameworks via nanotechnology in conjunction with stem cells is a new frontier for bone and tissue regeneration. Among the different scaffolds types, the ones that has fibrous architecture resembling the fibrillar structure of the ECM showed better support for the cell attachment and proliferation In this context, the electrospinning technique is an effective way to obtain fibers and membranes suitable for a tissue engineering scaffold. ${ }^{5}$ The electrospinning technique uses a high electrical potential applied to a polymer solution through a conductive capillary and a grounded collecting support. The increase in the voltage that suppress the surface tension of the solution causes an ejection of a charged jet that is stretched by the electric potential, the solvent is evaporated and fibers are deposited on the collector surface. ${ }^{6}$ In an attempt to improve the properties of such frameworks, it is assumed that providing specific nanostructured cell binding sites promotes cell differentiation. Therefore, in order to obtain scaffolds that mimic tissue and bone $\mathrm{ECM}$, it is essential to understand the effect of the parameters in the fiber manufacturing process of these frameworks when using the electrospinning technique. It is also necessary to correlate their microstructural features with the stem cells biocompatibility and the production of a mineralized matrix.

In this work, it was studied a different method of synthesis of PHB-HAp membranes obtained using the electrospinning technique. Usually, when polymers and ceramics membranes are obtained using this technique, there is a mixture of the components prior the electrospinning process. This often causes difficulties in the process (clog capillary, low dispersion of the ceramic particles, nonhomogeneous membranes). To avoid these problems, a simple PHB membrane was firstly obtain using electrospinning and two methods of impregnation in HAp solution were used thereafter. The result shows that the proposed methodology is effective to promote cell compatibility and growth. 


\section{Materials and methods}

\section{Membrane preparation}

We obtained polyhydroxybutyrate (PHB) and PHB-HAp composite membranes using the electrospinning technique. First, it was made a $0.5 \mathrm{mg} / \mathrm{mL}$ solution of PHB (Mw $2.3 \times 10^{5} \mathrm{~g} / \mathrm{mol}$; SigmaAldrich, Location) in chloroform (Synth, Location) using reflux at $70^{\circ} \mathrm{C}$ for 4 hours. N,N-Dimethylformamide (DMF, anhydrous, $99.8 \%$, Sigma-Aldrich) was added, and we magnetically stirred the mixture for 30minutes and applied ultrasound dispersion for 15 minutes. N,NDimethylformamide helps to reduce the evaporation of chloroform and aids in the formation of fibers during electrospinning. The electrospun PHB fibers were obtained using solution flow rates of 1.5, $2.0,3.0$ and $3.5 \mathrm{~mL} / \mathrm{h}$ controlled by a syringe pump (KDS100, KD Scientific), a distance of the needle-counter electrode of $140 \mathrm{~mm}$, a needle with a diameter of $1.2 \mathrm{~mm}$ and a voltage of $15 \mathrm{kV}$.

It was used two methods to obtain the PHB-HAp composite membranes:

A. Direct electro spinning: HAp (Flo Master 20.15.8F-3, F.J. Brodmann \& $\mathrm{Co}$ ) was added to the PHB solution previously prepared as described above and ultra sonified for 30 minutes. We evaluated fiber formation for three concentrations of HAp: 0.5, 0.2 and $0.1 \mathrm{mg} / \mathrm{mL}$ using the same distance and voltage of pure PHB fibers but flow rates of $3.0,3.5$ and $4.0 \mathrm{~mL} / \mathrm{h}$.

B. Impregnation: A dispersion of HAp in ethanol $(0.5,0.2$ and $0.1 \mathrm{mg} / \mathrm{mL}$ ) was obtained using an ultrasonic bath for 30 minutes, followed by the immersion of the PHB membranes previously obtained by electrospinning. The membrane was kept submerged in the HAp dispersion for 24hours, and then it was washed with water and ethanol to remove the excess Hap. Finally, we dried the membrane at room temperature.

\section{Characterization of the membranes}

It was used Scanning Electron Microscopy (SEM, JEOLJSM-6060) to characterize the morphology of the membranes that had been covered with carbon or gold to render them conductive. We measured the diameter of the fibers using Image $\mathbf{J}$ software as an aid to analyze the SEM images. We used $\mathrm{N}_{2}$ adsorption BET and BJH methods (Quanta chrome Nova 100e) to determine the specific surface area of the membranes and the pore size distribution. We used Raman spectroscopy (Renishaw in Via) to confirm the presence of HAp in the membranes using a laser wavelength of $532 \mathrm{~nm}$. To estimate the amount of HAp incorporated into the membranes and to evaluate the thermal stability of the membranes, it was performed thermogravimetry (TGA/SDTA 851e Mettler-Toledo) up to $1000^{\circ} \mathrm{C}$ using an atmosphere of synthetic air. We employed Dynamic Mechanic Analysis (TA Instruments, 2980) to determine the viscoelastic properties of the scaffolds. We used a dual-cantilever test with the following parameters: amplitude of $0.1 \%$, a frequency of $1 \mathrm{~Hz}$, a temperature range of $30-120^{\circ} \mathrm{C}$ and a heating rate of $2^{\circ} \mathrm{C} / \mathrm{min}$.

\section{Biocompatibility evaluation}

Cell culture: The bone marrow cells used in this work were obtained from the femur shaft of Wistar male rats. A suspension of the collected cells in Dulbecco's Modified Eagle's Medium (DMEM) was assembled, centrifuged $(400 \mathrm{~g})$ and resuspended in DMEM to separate and select the cells. We counted the cells and assessed their viability using the exclusion method with trypan blue in a Neubauer chamber. We conducted a cytotoxicity evaluation using NIH-3T3 mouse fibroblast cells. The cells were cultivated before each evaluation until confluence in a $75 \mathrm{~cm}^{2}$ cell culture flask containing DMEM, supplemented with $10 \%$ Fetal Bovine Serum (FBS), gentamicin $(0.025 \mathrm{~g} / \mathrm{L}$ and streptomycin/penicillin $(0.1 \mathrm{~g} / \mathrm{L})$ in a humid atmosphere at $37^{\circ} \mathrm{C}$ with $5 \% \mathrm{CO}_{2}$.

Cytotoxicity evaluation: The cytotoxicity of the scaffolds was evaluated according to the ISO 10993-5 using an MTT assay to evaluate the mitochondrial function. The membranes, after being sterilized with ultraviolet light, were immersed in DMEM for 24,72 or 168 hours and incubated with stirring in a humid atmosphere at $37^{\circ} \mathrm{C}$ with $5 \% \mathrm{CO}_{2}$. DMEM was used as a negative control, and copper sulfate $\left(0.1 \mathrm{~g} \cdot \mathrm{mL}^{-1}\right)$ in DMEM was used as a positive control.

Scaffold mineralization: To evaluate the mineralization of the scaffolds, we used an alizarin red-based assay. To quantify the mineralization among the different periods, the alizarin red was removed using acetic acid $(10 \%)$ in DMSO. We used a blank test for each period and a positive control test using tibia from a stem cell rat donor. We analyzed the quantitative data using ANOVA and Tukey's test. Results with $p<0.05$ were considered to be statistically significant.

\section{Results and discussion}

\section{Membrane characterization}

The synthesized membranes ought to have characteristics such a porous structure and a surface area that is adequate to enable cell adhesion, proliferation and differentiation. When we analyzed the morphology of the PHB membranes samples obtained by electrospinning (Figure 1), we observed the formation of a weblike structure composed of randomly oriented fibers. It was possible to observe the effect of the applied flow rate on the membrane morphology; an increased flow rate corresponds to a diminished presence of beads and a smaller apparent mean bead diameter. The membranes produced using a flow rate of $3.5 \mathrm{~mL} / \mathrm{h}$ exhibited fibers $0.552 \pm 0.152 \mu \mathrm{m}$ in diameter. We selected this membrane for further analyses (i.e., biocompatibility tests) because it had no beads and its fibers were the thinnest. The incorporation of HAp in the PHB solution during the electrospinning process resulted in fibers with a mean diameter of $0.627 \pm 0.190 \mu \mathrm{m}$ containing agglomerated HAp beads approximately $5.58 \pm 2.05 \mu \mathrm{m}$ in diameter (Figure 2). However, when we used the immersion technique, the PHB membranes included smaller agglomerated HAp beads approximately $20 \mathrm{~nm}$ in diameter (Figure 3), and the fiber diameter was approximately $0.668 \pm 0.134 \mu \mathrm{m}$. To investigate the biocompatibility of the membranes, we selected three scaffolds: one made of pure PHB fibers and two composites with HAp ( $\mathrm{C} 1$ and $\mathrm{C} 2)$. In the $\mathrm{C} 1$ membrane, the HAp beads were randomly dispersed in the organic matrix (PHB); in the $\mathrm{C} 2$ membrane, the nanostructured HAp crystals covered the surface of the PHB fibers. The presence of HAp in the surface of the fibers was confirmed via EDS analysis. The HAp presented a $\mathrm{Ca} / \mathrm{P}$ molar ratio of 1.67 . The composite membrane obtained via the immersion presented a microstructure of HAp dispersed in the PHB matrix with a mean diameter of approximately $64 \mathrm{~nm}$. Meanwhile, in the $\mathrm{C} 2$ membranes obtained by immersing the PHB membrane in the supernatant of the HAp suspension, the HAp particles remained on the surface of the fibers with a smaller mean diameter of approximately $40 \mathrm{~nm}$. When one compares the PHB and the HAp composite membranes, it is possible to observe a significant change in the scaffold structure, particularly 
in the amount of fibers per volume of the electrospun matrix and the consequent increase in the pore size among the fiber structure.
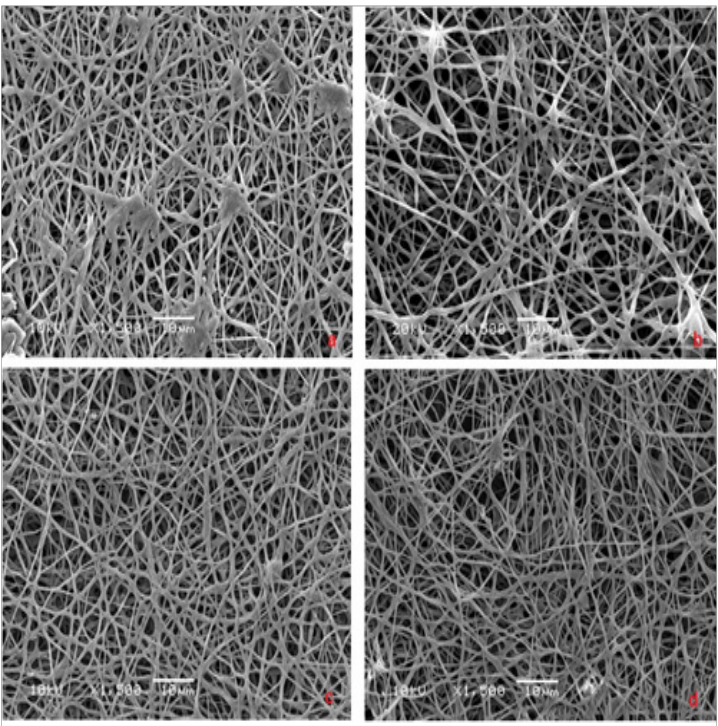

Figure I SEM images of PHB membranes obtained using a flow rate of a) I.5, b) 2.0 , c) 3.0 e d) $3.5 \mathrm{~mL} / \mathrm{h}$
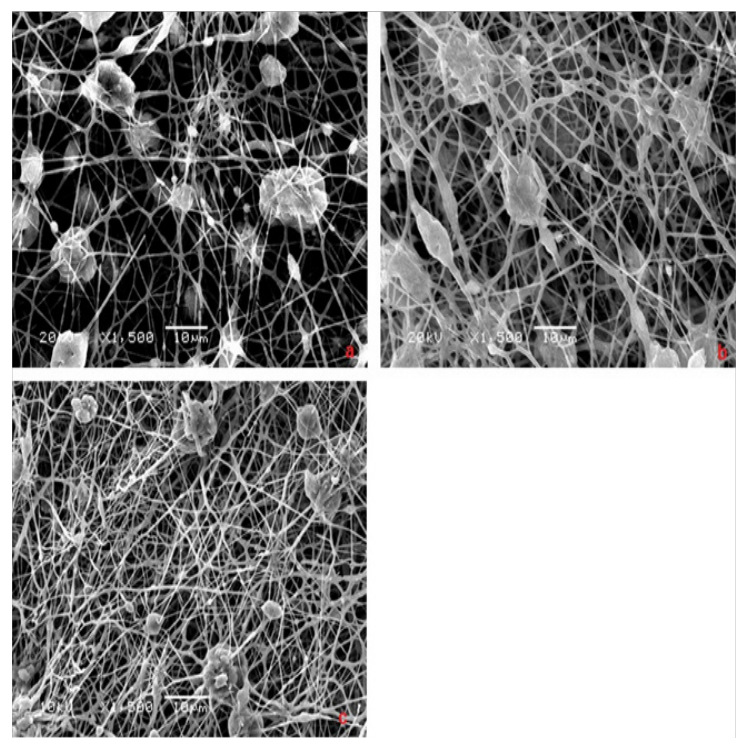

Figure 2 SEM images of PHB+HAp composite fibers: a) 3.0; b) 3.5 and c) $4.0 \mathrm{~mL} / \mathrm{h}$

The pore size distribution and specific surface area (Table 1) indicate that the membranes are mesoporous, with pore diameters ranging between 2 and $50 \mathrm{~nm}$. In the case of the $\mathrm{C} 1$ and $\mathrm{C} 2$ composites, the presence of HAp increased the total pore volume and its diameter compared with the data of pure PHB membranes, independent of the method of HAp incorporation. The presence of mesopores enhances the adsorbent characteristics of drugs or specific molecules that can direct regeneration and modulate the immunological response of this biomaterial. The specific surface was significantly affected by the presence and amount of HAp in the surface of the fibers, an effect that is likely due to the porous structure of the HAp particles.

The Raman spectra of the membranes in Figure 4 indicate the organic fraction of PHB and the presence of HAp. The PHB characteristic Raman bands are located at 433,599, 840 and $1058 \mathrm{~cm}^{-}$ 1. The Raman shift band of HAp at $430 \mathrm{~cm}^{-1}$ corresponds to the axisymmetric deformation of $\mathrm{PO}_{4}$, and the shifts between 550 and $600 \mathrm{~cm}^{-1}$ are superimposed with the Raman shifts of PHB; these shifts are related to the deformation of - $\mathrm{CCO}-$ and $-\mathrm{C}-\mathrm{CH}_{3}$ groups. ${ }^{7}$ It is also possible to observe that the $\mathrm{C} 2$ scaffold has lower-intensity HAp peaks compared with those of the $\mathrm{C} 1$ scaffold. This result may be related to a lower concentration of HAp in these membranes. Indeed, when the thermogravimetric results are analyzed (Figure 5), the decomposition of PHB and the remaining mass related to residual Hap are notable. The scaffold composed of pure PHB is practically completed decomposed at $500^{\circ} \mathrm{C}$. The $\mathrm{C} 1$ and $\mathrm{C} 2$ composite membranes present approximately 35 and $11 \%$ HAp by mass, respectively. Up to $200^{\circ} \mathrm{C}$, none of the membranes present a significant weight loss, indicating that the majority of the solvents used during the electrospinning process were evaporated during the synthesis. The addition of HAp in PHB membranes yielded a decrease in the viscoelastic properties of the PHB scaffolds, independent of the quantity of HAp present in the membrane structure. PHB has a storage modulus of 424MPa; the $\mathrm{C} 1$ and $\mathrm{C} 2$ composites membranes have moduli of 26 and $28 \mathrm{MPa}$, respectively.

This effect can be related to the plasticizing effect of HAp when it is incorporated in polymers and also the interaction of carbonyl groups of PHB with $\mathrm{Ca}^{+2}$ from the HAp, which creates intermolecular interactions that reduce the visco elasticity of such polymers. ${ }^{8,9}$

Table I Specific surface area, volume and diameter of mesopores in the synthesized membranes

\begin{tabular}{llll}
\hline Membrane & $\begin{array}{l}\text { Specific } \\
\text { surface area } \\
\mathbf{S}_{\text {BET }}\left(\mathbf{M}^{2} / \mathbf{G}\right)\end{array}$ & $\begin{array}{l}\text { Mesopores } \\
\text { volume(Cm }\end{array}$ & $\begin{array}{l}\text { Mesopores } \\
\text { diameter(Nm) }\end{array}$ \\
\hline $\mathrm{PHB}$ & 2,5 & 0,015 & 4,4 \\
$\mathrm{Cl}$ & 7,1 & 0,047 & 12,3 \\
$\mathrm{C} 2$ & 4,9 & 0,048 & 12,5 \\
\hline
\end{tabular}

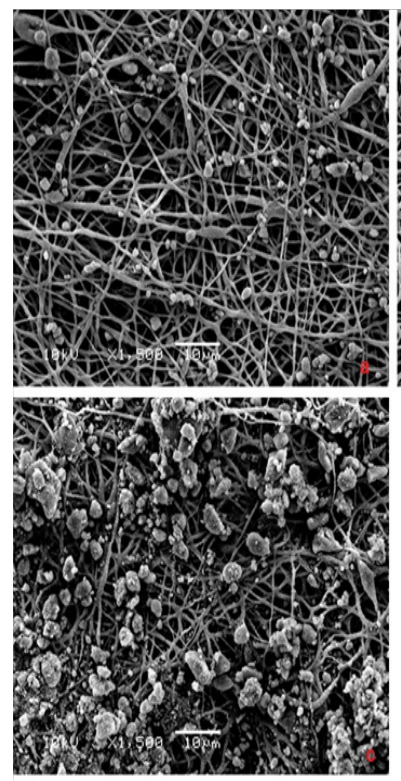

Figure 3 SEM images of $\mathrm{PHB}+\mathrm{HAp}$ composite fibers obtained by immersion method. Effect of HAp concentration: a) $0,1 \mathrm{mg} / \mathrm{mL}$, b) $0,2 \mathrm{mg} / \mathrm{mL}$ e c) $0,5 \mathrm{mg} /$ $\mathrm{mL}$. 


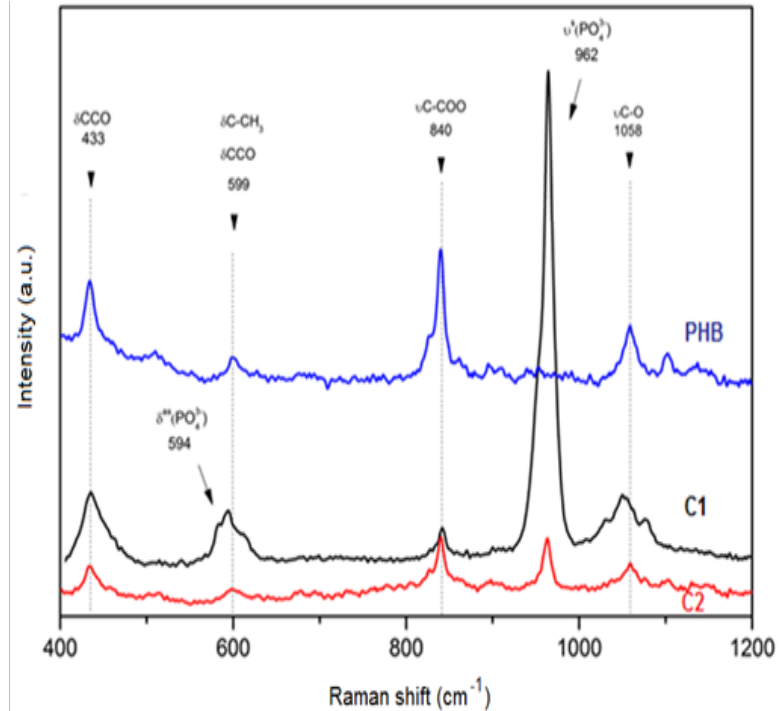

Figure 4 Raman spectra of PHB and PHB-HAp composite scaffolds. $\delta$, deformation, $\mathrm{v}$, stretching, $\mathrm{s}$, symmetric, as, axisymmetric

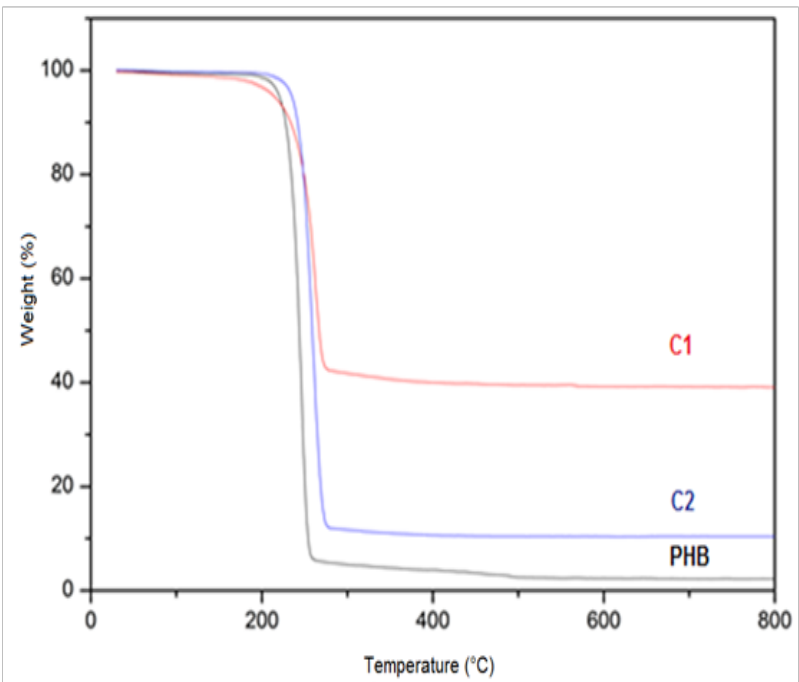

Figure 5 Thermogravimetric results of $\mathrm{PHB}$ and $\mathrm{PHB}+\mathrm{HAp}$ composites in synthetic air

\section{Biocompatibility assay}

It was conducted a phenotypic characterization of the bone marrow cells using flow cytometry. Our findings revealed that $6.55 \%$ of cells presented the CD45 marker, $1.77 \%$ of cells presented the CD3 marker, $4.82 \%$ of cells presented the CD49b marker and $23.55 \%$ of cells presented the CD90 marker. According to ISO 10993-5 and evaluation of medical devices In vitro materials that present a cellular viability above $80 \%$ are considered to be low or non-cytotoxic, and they are good candidates for medical use. The cytotoxicity results for 3, 7 and 14 days presented in Figure 7 show that the cellular viability was higher than $79 \%$ for all of the scaffolds analyzed. The $\mathrm{C} 1$ composite presented the highest viability among of the synthesized membranes, with values of $97 \%$ for 3 days and up to $100 \%$ after 14 days. In general, implanted materials have an immunological response that is caused by the material itself or the surgical procedure. Upon this response, the implant will be successful in its function or it will be rejected or encapsulated. The investigated scaffolds exhibited a mild cytotoxic response that could be beneficial in the cicatrizing process and remodeling the tissue surrounding the implantation area. Recent studies showed that stem cells are attracted to places that present a tissue injury by the presence of chemokines that module the local inflammatory response. ${ }^{10,11}$
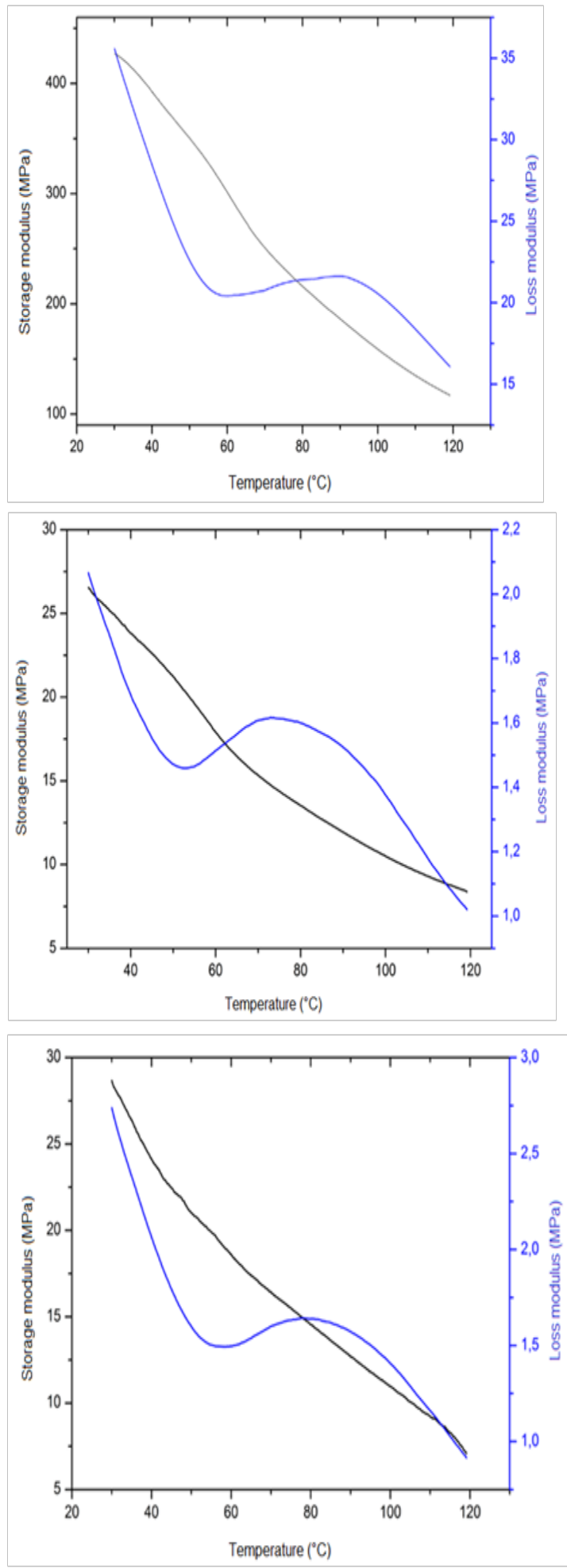

Figure 6 DMA curve of the scaffolds, representing the storage and loss modules as a function of the temperature: (A) PHB, (B) $\mathrm{Cl}$, ( C) $\mathrm{C} 2$ 


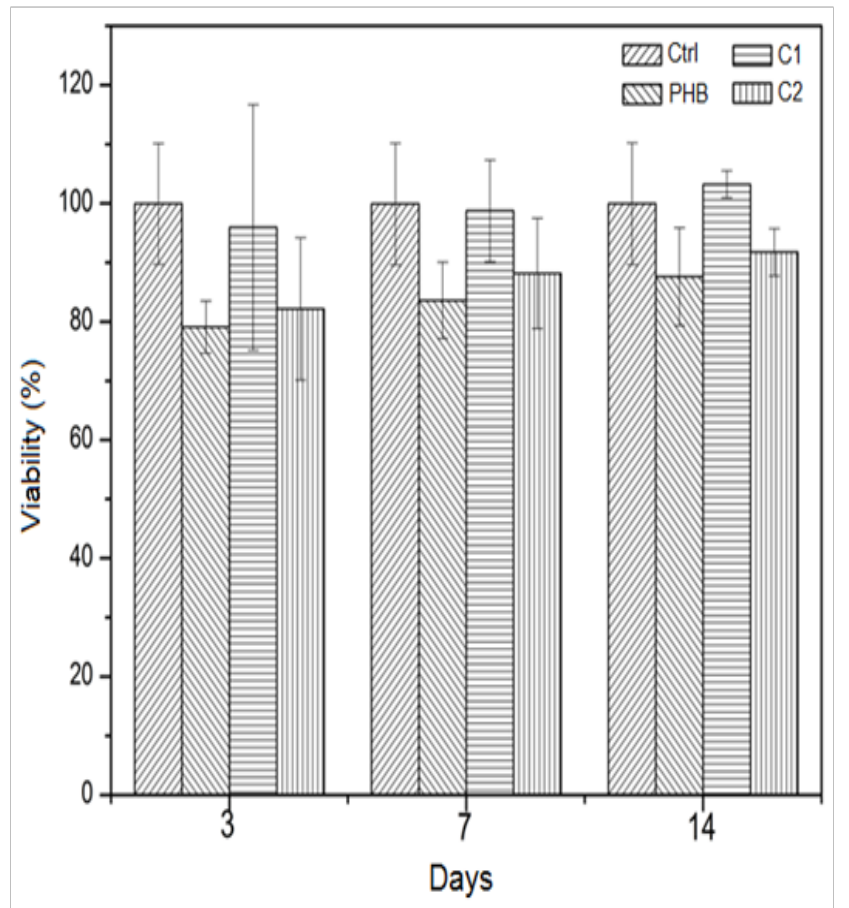

Figure 7 Cytotoxicity evaluation of PHB and PHB+HAp scaffolds

Cellular adhesion is related to the capacity of regeneration and remodeling of soft and hard tissues. In the case of the proposed membranes, it is expected that the cells adhere to them to promote their growth and then tissue regeneration. The capacity of the cells to adhere and proliferate in the surface of a material is the first phase of interaction material-cells. ${ }^{12}$ we accordingly analyzed the adhesion and proliferation of mesenchymal stem cells in the scaffolds produced by electrospinning. Figure 8 shows a graph of the proliferation of the scaffolds over different time periods. Since adherence and proliferation are features that are closely linked in the cell-material interaction, they will be discussed together to better understand the mechanisms involved. It was observed that the cells proliferated in all periods that we evaluated, which is indicative of excellent cellular activity. When comparing the proliferation between days for each group, we found a statistical difference in the control group on day 3 compared with days 7 and 14. On the other hand, the PHB group exhibited a significant difference in the proliferation on days 3 and 7 compared with day 14. Both HAp composite membranes only exhibited a statistical difference on day 3 compared with days 7 and 14. However, when the groups were compared among themselves in each period, we noted a difference in the proliferation of PHB group on day 3 compared with the control groups on day 7 and both frameworks and controls on day 14. In other periods and groups, no significant differences were observed. When one looks at the graph of proliferation, it is obvious that the PHB group exhibits low proliferation for the time period between 3 and 7 days. These curves can be explained by the characteristics of the cell and its interactions with the surface of PHB framework. Also, there was a decrease in the control group proliferation between days 7 and 14 .

In the case of the control group, the decreased of the proliferation rate can be attributed to the high cell density found on day 7 , which is best seen in the micrograph of control the stained cells on day 7. This high density may have contributed to the reduction of cell proliferation at the well surface on the culture plate, in which one can observe a confluence of cells. Therefore, the lack of space for the cells to grow may have caused the decrease in mitotic activity. Mitotic activity is a mechanism found in eukaryotic cells that helps maintain tissue equilibrium, enable organized growth and facilitate the avoidance of uncontrolled growth and the emergence of cancer, for example. One of the factors that triggers the mechanism is the inhibition of cell proliferation by contact between the cells when they are in conditions characterized by high density.

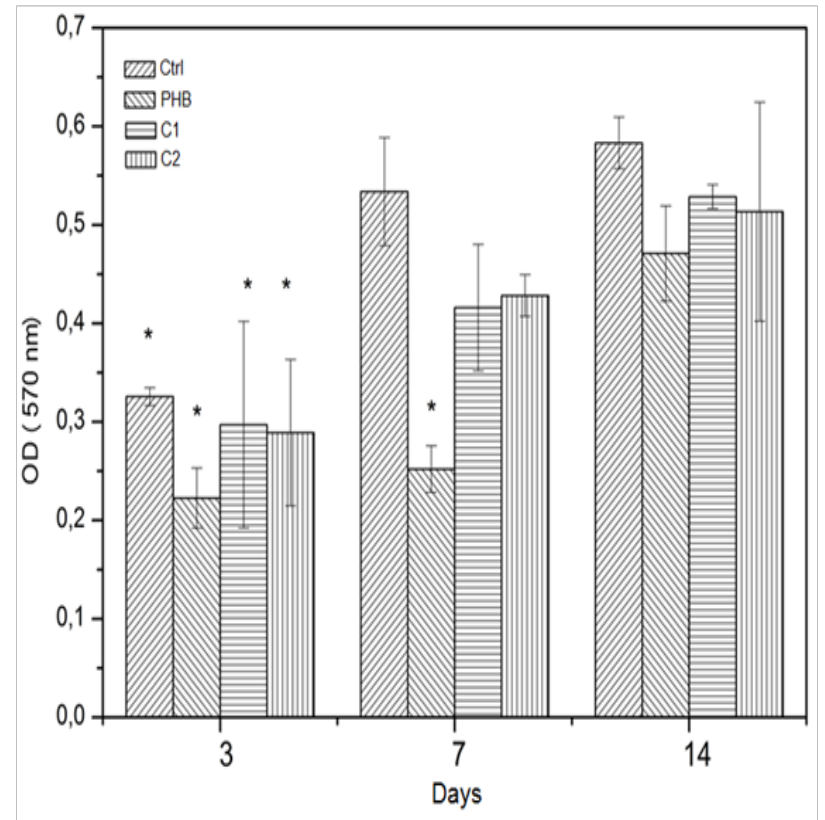

Figure 8 Cellular proliferation rate: optical density as a function of cultivation time $(* \mathrm{p}<0.05 ; \mathrm{n}=3)$

Consequently, for the PHB group, the surface of this polymer may have contributed to the decrease in cell adhesion. Likely due to the PHB hydrophobicity, there was lower cell adhesion and therefore a lower proliferation rate during the initial periods. However, we observed that the cells proliferated until day 14. Studies have shown that the balance between hydrophilic and hydrophobic groups contributes to better cell adhesion. ${ }^{13}$ Therefore, with the addition of the $\mathrm{HAp}$ in the composites $\mathrm{C} 1$ and $\mathrm{C} 2$ composites; there was a reduction in the PHB hydrophobicity due to the contribution of the HAp hydrophilicity, which contributed to better cell adhesion. However, the cell proliferation remains discrete for the $\mathrm{C} 1$ and $\mathrm{C} 2$ groups during the studied periods, but it had a constant rate. This simple proliferation of cells on scaffolds $\mathrm{C} 1$ and $\mathrm{C} 2$ can be linked to the commitment of stem cells in the osteoprogenitor lineage.

By the third day of culture, both groups had roughly the same cell density and did not produce a mineralized matrix. After the third day, the formation of a mineralized matrix in the frameworks $\mathrm{C} 1$ and C2 was initiated, and there was a decrease in the cell proliferation rate compared with the control. The decrease in proliferation rate may be associated with the loss of the self-renewal characteristics, typical of stem cells, leading to a decrease in the kinetics of division of adherent cells in the composites. This effect was not observed in the PHB membranes because the cells, after day 7, exhibited a high proliferation rate. Figures 9-11 show images obtained using optical microscopy of adherent cells in the scaffolds and the control group 
on days 3, 7 and 14, respectively. When analyzing these images, it appears that the cells in the membranes have similar morphologies as the ones of the control group with the characteristics of adhered cells (i.e., a starry morphology). Also, we observed cell-cell interactions that were sprawling over the surface of the scaffolds due to cytoplasmic extensions (indicated with black arrows). We observed, especially in $\mathrm{C} 1$ composite membrane, that the cells were located within the matrix. As seen from the SEM images, the HAp dispersed in the matrix decreased the number of fibers and resulted in a larger space between them. Consequently, there was an increase in the pore size between fibers. This layered structure allows cells to infiltrate the scaffold matrix. The occasionally anchoring of the cells along the fibers (indicated with white arrows) is able notable.
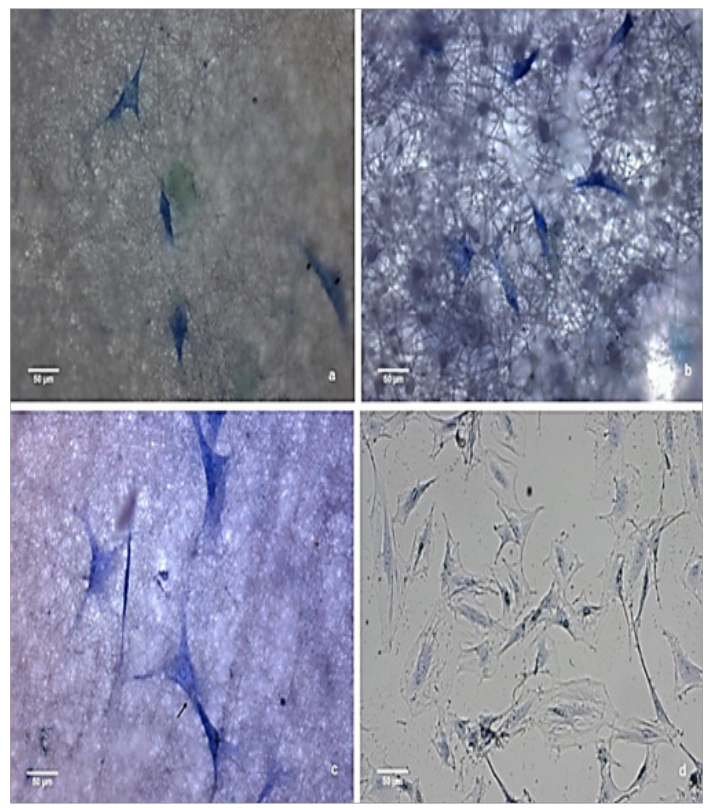

Figure 9 Stem cell culture adhere to the membranes after three days: (A) $\mathrm{PHB}(\mathrm{B}) \mathrm{Cl}$ (C) $\mathrm{C} 2$ e (D) control
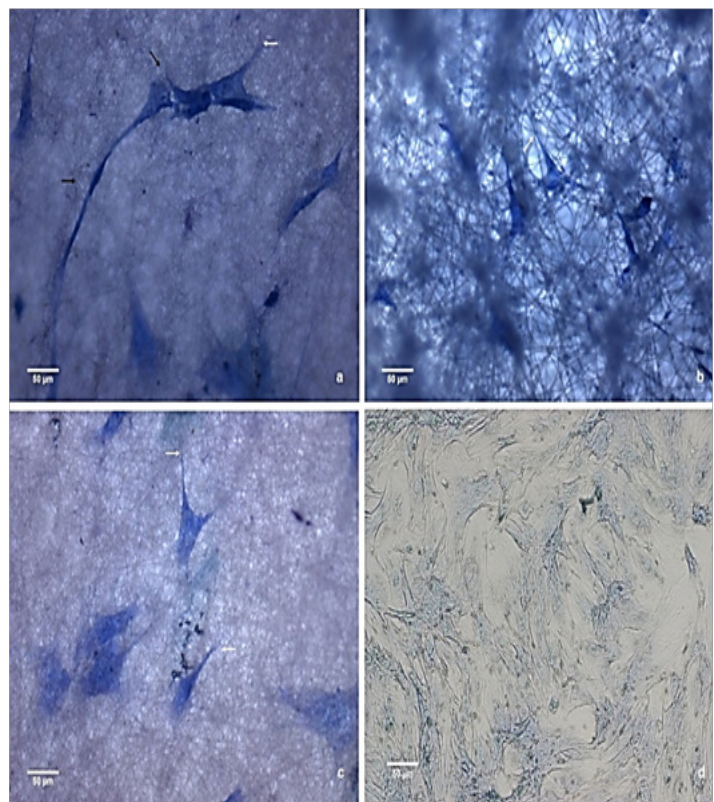

Figure 10 Stem cell culture adhere to the membranes after seven days: $(A)$ $\mathrm{PHB}(\mathrm{B}) \mathrm{Cl}$ (C) $\mathrm{C} 2$ e (D) control
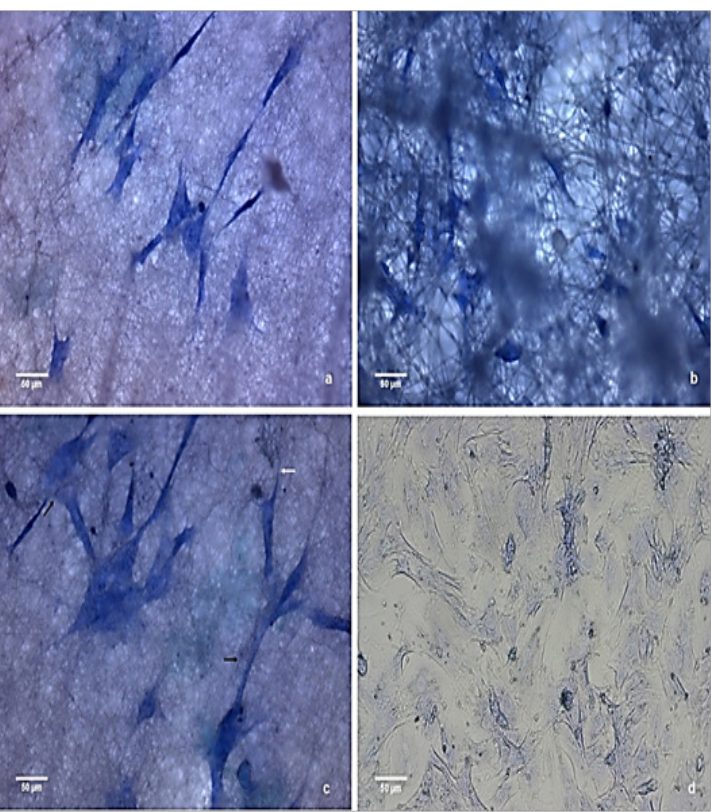

Figure I I Stem cell culture adhere to the membranes after fourteen days: (A) PHB (B) Cl (C) C2 e (D) control

Similar results were obtained Ito et al. ${ }^{14}$ who manufactured electrospun membranes of poly (3-hydroxybutyrate -co -3hydroxyvalerate) (PHBV) and produced a composite with hydroxyapatite via the immersion technique in a simulated body fluid. These authors observed that the hydrophobicity of the nanofibers decreased after the deposition of HAp and that there was an increase in the specific surface area of the composite HAp membrane. Consequently, there was an increase in cell adhesion, although the cell adhesion was not significantly affected by the combination with HAp.

Figure 12 shows the results of optical density values of the scaffolds investigated as a function of time compared with the optical density of cortical bone used as a positive control of a mineralized matrix. The production of mineralized matrix is an indicator of bone cell differentiation, and alizarin is used to confirm bone cell differentiation via a color indication (red) Birmingham, Kyllönen \& Uddin ${ }^{15-17}$ due to its complexation with calcium found in the HAp when observed using optical microscopy. Based on Figure 12 all of the membranes and the control (mesenchymal cells) were significantly different compared with the positive control (cortical bone). However, the $\mathrm{C} 1$ and $\mathrm{C} 2$ groups exhibited an increase in mineralization beginning on day 7 , indicating that the cells were producing a mineralized matrix on the scaffolds. Comparing the control group and the PHB membrane, the cells maintained a basal level at all time periods, which is not an indicator of low production of the mineralized matrix but instead due to the calcium found in the cells because of their normal metabolism.

In the same figure, one can compare the control group and the membranes and observe the formation of mineralized matrix scaffolds in the $\mathrm{C} 1$ and $\mathrm{C} 2$ groups. On day 3, both groups exhibited basal level activity; on day 7, it was possible to observe the production of mineralized matrix on the $\mathrm{C} 1$ and $\mathrm{C} 2$ scaffolds. In the case of the $\mathrm{C} 1$ composite compared with the control group on day 7 , there was a significant difference in the production of the mineralized matrix. The same was not true when comparing the $\mathrm{C} 1$ composite with the $\mathrm{C} 2$ composite and PHB membranes over the same period. However, when compared on day 3 , all groups showed significant differences; a 
significant difference was noted on day 14 between the control group and the PHB membrane. On day 14, the $\mathrm{C} 1$ composite differed in the production of mineralized matrix compared with both the control and PHB groups in all periods, but there was no statistical difference when we compared the $\mathrm{C} 2$ and $\mathrm{C} 1$ groups on day 7 . The $\mathrm{C} 2$ membrane on day 14 also exhibited a significant difference compared with the control and PHB groups. However, on days 3 and 7, there was no significant difference when the $\mathrm{C} 2$ membrane was compared with the control group and the PHB. On days 7 and 14, a significant difference was not found when we compared the frameworks of $\mathrm{C} 1$ and $\mathrm{C} 2$. It is evident that the addition of HAp and the fiber structure contribute to the induction of stem cell adhesion and proliferation and promote the mineralization of membranes. These adhesion control mechanisms highlight the importance of the microenvironment in the regulation of stem cell differentiation. Indeed, the development of an ECM during osteogenesis is essential for bone development. Studies in the literature that are consistent with the findings of this study demonstrate that the increased roughness on the polymer surface (i.e., the larger range of height irregularities) leads to decreased osteoblast proliferation and increased osteogenic differentiation. ${ }^{18}$ The existence of HAp in the polymeric matrix can accelerate the In vitro mineralization kinetics of the ECM. ${ }^{19}$ Other evidences with respect to microstructures and nanostructures indicate that these structures increase the specific surface area of the membranes and promote interaction with growth factors and consequently trigger the induction of osteo-progenitor cells undifferentiated into the osteogenic lineage by increasing the expression of osteocalcin, a specific marker involved in bone mineralization control. ${ }^{19,20}$

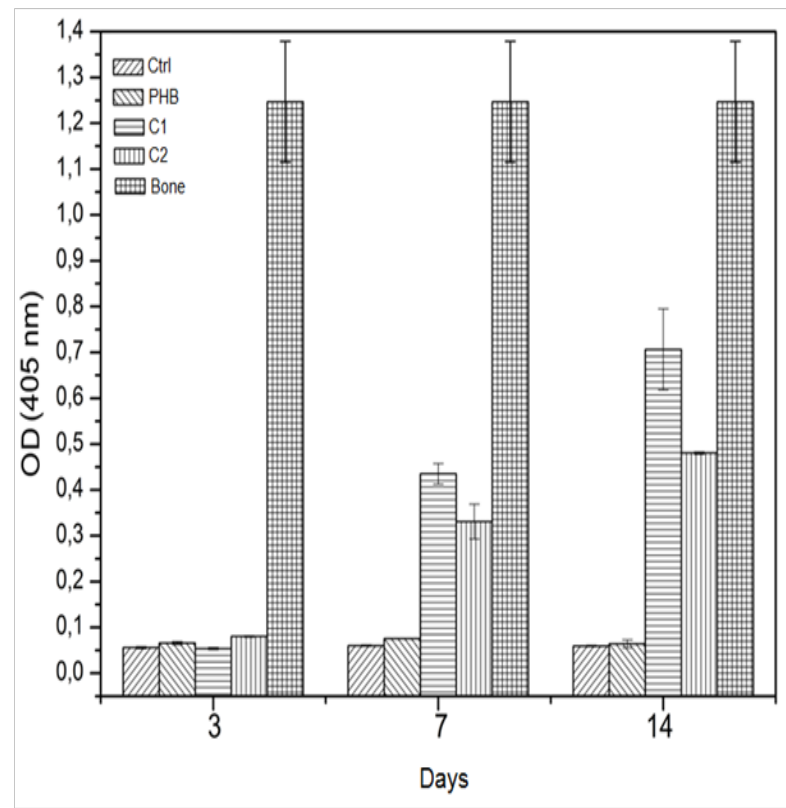

Figure 12 Optical density values in the synthesized membranes as function of time, compared to the optical density of cortical bone $(n=3, p<0.05)$

\section{Conclusion}

It was possible to produce microstructured and nanostructured PHB and HAp membranes using the electrospinning technique. These fibers had different structures and diameters: the PHB membrane had a diameter of $0.55 \pm 0.15 \mu \mathrm{m}$, the HAp composite membrane obtained with the incorporation of the particles during the electrospinning process $(\mathrm{C} 1)$ had a diameter of $0.62 \pm 0.19 \mu \mathrm{m}$ and the membrane obtained using the immersion method of the supernatant of a HAp solution in a electrospun PHB membrane (C2) had a diameter of $0.66 \pm 0.13 \mu \mathrm{m}$. There was an increase in the surface area of the $\mathrm{C} 1$ and $\mathrm{C} 2$ composite membranes of about $200 \%$ and $100 \%$, respectively, compared with the PHB framework. We also observed an increase of $200 \%$ in the diameters of the mesopores of both composites compared with the PHB membrane.

It was possible to evaluate the HAp content in the membranes (45 to $16 \%$ by weight of the $\mathrm{C} 1$ and $\mathrm{C} 2$ membranes, respectively); the $\mathrm{C} 2$ membrane is more interesting for the manufacture of composites for use in bone regeneration due to the smaller amount of HAp used in the production of the membrane and the fact that it produces a mineralization that is effective as the one observed in the C1 membrane. All scaffolds exhibited high biocompatibility, about $80 \%$, and promoted adhesion and proliferation of mesenchymal stem cells. The presence of a mineral phase of HAp in the composite membranes favored the production of a mineralized cell matrix. The deposition of a mineralized matrix and cellular activity is indicative of differentiation of mesenchymal stem cells into the osteogenic lineage. The scaffolds produced have osteoconductive characteristics and osteo activity, which makes them excellent candidates for scaffolds used in bone regeneration that are not exposed to excessive forces.

\section{Acknowledgments}

The authors would like to thank the U.S. Office of Naval Research Global (award No N62909-15-1-N081) for the financial support.

\section{Conflict of interest}

The author declares that there is no conflict of interest.

\section{References}

1. Pohl S, Haas R, Tangl S, et al. A description of the sequence of longterm behavior of autogenous boneblock in maxillary sinus augmentation in sheep: Additional morphologic and histomorphometric evidence. Oral Surgery, Oral Medicine, Oral Pathology and Oral Radiology. 2018;125(1):6-13.

2. Correia DM, Ribeiro CR, Ferreira JCC, et al. Influence of Electrospinning Parameters on Poly (hydroxybutyrate) Electrospun Membranes Fiber Size and Distribution. Polymer Eng and Sci. 2013;54(7)1608-1617.

3. Nikolaou VS, Giannoudis PV. History of osteochondral allograft transplantation. Injury. 2017;48(7):1283-1286.

4. Boisseau P, Loubaton B. Nanomedicine, nanotechnology in medicine. Comptes Rendus Physique. 2011;12(7):620-636.

5. Sadat-Shojai M, Khorasani MT, Jamshidi A. A new strategy for fabrication of bone scaffolds using electrospun nano-HAp/PHB fibers and protein hydrogels. Chemical Engineering Journal. 2016;289(1):38-47.

6. Liu M, Duan XP, Li YM, et al. Electrospun nanofibers for wound healing. Materials Science and Engineering: C. 2017;76(1):1413-1423.

7. Furukawa T, Sato H, Murakami R, et al. Raman microspectroscopy study of structure, dispersibility, and crystallinity of poly (hydroxybutyrate)/poly (L-lactic acid) blends. Polymer. 2006;47(9):3132-3140.

8. Chen Y, Mak AFT, Li JS, et al. Formation of apatite on poly (alpha-hydroxy acid) in an accelerated biomimetic process. J of Biomed Mat Research Part B -Applied Biomaterials. 2005;73(1):68-76.

9. Ngiam M, Liao SS, Patil AJ, et al. The fabrication of nano-hydroxyapatite on PLGA and PLGA/collagen nanofibrous composite scaffolds and their effects in osteoblastic behavior for bone tissue engineering. Bone. 2009;45(1):4-16. 
10. Dazzi F, Lopes L, Weng L. Mesenchymal stromal cells: a key player in innate tolerance'? Immunology. 2012;137(3):206-213.

11. Scheibe F, Ladhoff J, Huck J, et al. Immune effects of mesenchymal stromal cells in experimental stroke. J of Cerebral Blood Flow and Metabolism. 2012;32(8):1578-1588.

12. El-Amin SF, Lu HH, Khan Y, et al. Extracellular matrix production by human osteoblasts cultured on biodegradable polymers applicable for tissue engineering. Biomaterials. 2003;24(7):1213-1221.

13. Olmedilla MP, Garcia-Giralt N, Pradas MM, et al. Response of human chondrocytes to a non-uniform distribution of hydrophilic domains on poly (ethyl acrylate-co-hydroxyethyl methacrylate) copolymers. Biomaterials. 2006;27(7):1003-1012.

14. Ito $\mathrm{Y}$, Hasuda $\mathrm{H}$, Kamitakahara M, et al. A composite of hydroxyapatite with electrospun biodegradable nanofibers as a tissue engineering material. J of Biosci and Bioeng. 2005;100(1):43-49.

15. Birmingham E, Niebur GL, Mchugh PE, et al. Osteogenic Differentiation of Mesenchymal Stem Cells Is Regulated by Osteocyte and Osteoblast Cells in a Simplified Bone Niche. European Cells \& Materials. 2012;23:13-27.
16. Kyllonen L, Haimi S, Mannerstrom B, et al. Effects of different serum conditions on osteogenic differentiation of human adipose stem cells In vitro. Stem Cell Research \& Therapy. 2013;4(1):1-17.

17. Uddin SMZ, Qin YX. Enhancement of Osteogenic Differentiation and Proliferation in Human Mesenchymal Stem Cells by a Modified Low Intensity Ultrasound Stimulation under Simulated Microgravity. Plos One. 2013;8(9):73914.

18. Barbieri D, Yuan H, Luo X, et al. Influence of polymer molecular weight in osteoinductive composites for bone tissue regeneration. Acta Bio materialia. 2013;9(12):9401-9413.

19. Fricain JC, Schlaubitz S, Le Visage C, et al. A nano-hydroxyapatite-Pullulan/dextran polysaccharide composite macroporous material for bone tissue engineering. Biomaterials. 2013;34(12):2947-2959.

20. International Organization for Standardization ISO10993-5. Biological evaluation of medical devices. Part 5: Tests for cytotoxicity: In vitro methods. Switzerland; 1999. 